\title{
Highly suspected primary intraocular lymphoma in a patient with rheumatoid arthritis treated with etanercept: a case report
}

\author{
Woo Keun Song, Ah Ran Cho and Young Hee Yoon
}

\begin{abstract}
Background: To describe a case of highly suspected primary intraocular lymphoma (PIOL) in a patient using etanercept for the treatment of rheumatoid arthritis.

Case presentation: A 50-year-old female patient presented with decreased vision in her left eye that lasted for a week. She had a 15-year history of seropositive rheumatoid arthritis (RA), and had been taking weekly etanercept for the preceding 8 months. Funduscopic examination and SD-OCT showed a swollen ellipsoid zone (EZ) and a retinal pigment epithelium (RPE) irregularity of the right eye. We also noted EZ disruption and a RPE irregularity in the left eye. As subretinal infiltration was aggravated in the right eye after the initial treatment, we completed a vitrectomy. Vitreous cytology revealed PIOL with positive CD20 immunostaining. She was treated with serial intravitreal methotrexate injections and systemic chemotherapy. After the treatment, subretinal infiltration and subRPE deposits were decreased in the right eye with no evidence of recurrence in either eye.

Conclusions: This case suggests a potential relationship between immunosuppression with anti-TNFa medication, and increased risk for lymphoma, especially in patients with underlying rheumatologic disorders and especially in patients with suspected chronic refractory uveitis.
\end{abstract}

Keywords: Primary intraocular lymphoma, Etanercept, Methotrexate, Spectralis-domain optical coherence tomography

\section{Background}

Tumor necrosis factor alpha (TNF $\alpha)$ plays a role in the normal inflammatory response and immune reaction. Although the safety and efficacy of anti-TNF $\alpha$ agents have already been established, the risk of lymphoma appears higher in patients treated with immune-modulating agents $[1,2]$. This case report describes a patient with highly suspected primary intraocular lymphoma (PIOL), possibly associated with use of the approved anti-TNFo agent etanercept for treatment of rheumatoid arthritis (RA) [3].

\footnotetext{
* Correspondence: yhyoon@amc.seoul.kr

Department of Ophthalmology, College of Medicine, University of Ulsan, Asan Medical Center, 88 Olympic-ro 43-Gil, Songpa-gu, Seoul 05505, South Korea
}

(c) The Author(s). 2018 Open Access This article is distributed under the terms of the Creative Commons Attribution 4.0 International License (http://creativecommons.org/licenses/by/4.0/), which permits unrestricted use, distribution, and reproduction in any medium, provided you give appropriate credit to the original author(s) and the source, provide a link to the Creative Commons license, and indicate if changes were made. The Creative Commons Public Domain Dedication waiver (http://creativecommons.org/publicdomain/zero/1.0/) applies to the data made available in this article, unless otherwise stated.

\section{Case presentation}

A 50-year-old female patient presented with a one-week history of decreased vision in her left eye. She had a 15-year history of seropositive RA treated with methotrexate and deflazacort. Because of an unsatisfactory response to those regimens, she was initiated on $25 \mathrm{mg}$ per week of etanercept 8 months prior to presentation.

On presentation, her best-corrected visual acuity (BCVA) was 0.8 OD and 0.1 OS. There were no cells in the anterior chamber of either eye. Funduscopic examination showed granular infiltration at the temporal macula in the right eye and the foveal area in the left eye (Fig. 1a). Spectral-domain optical coherence tomography showed a swollen ellipsoid zone and retinal pigment epithelium (RPE) irregularities in the right eye and an ellipsoid zone disruption and RPE irregularity in the left 


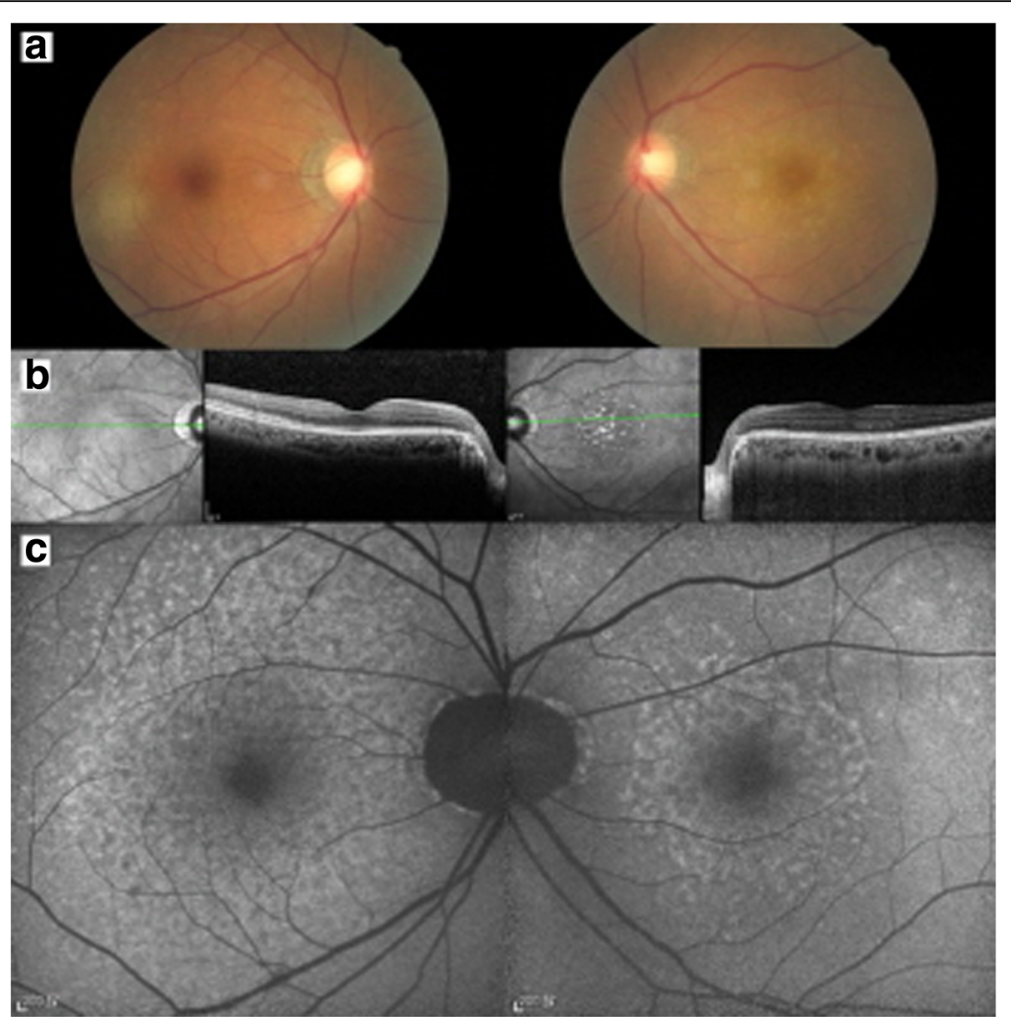

Fig. 1 Initial ophthalmologic findings. a Funduscopic examination showed a granular infiltration temporal to the macula in the right eye and in the whole macular area of the left eye. $\mathbf{b}$ Spectral-domain optical coherence tomography showed a swollen ellipsoid zone and a retinal pigment epithelium irregularity in the right eye and an ellipsoid zone disruption in the left eye. c Fundus autofluorescence showed parafoveal granular hyperautofluorescence in both eyes

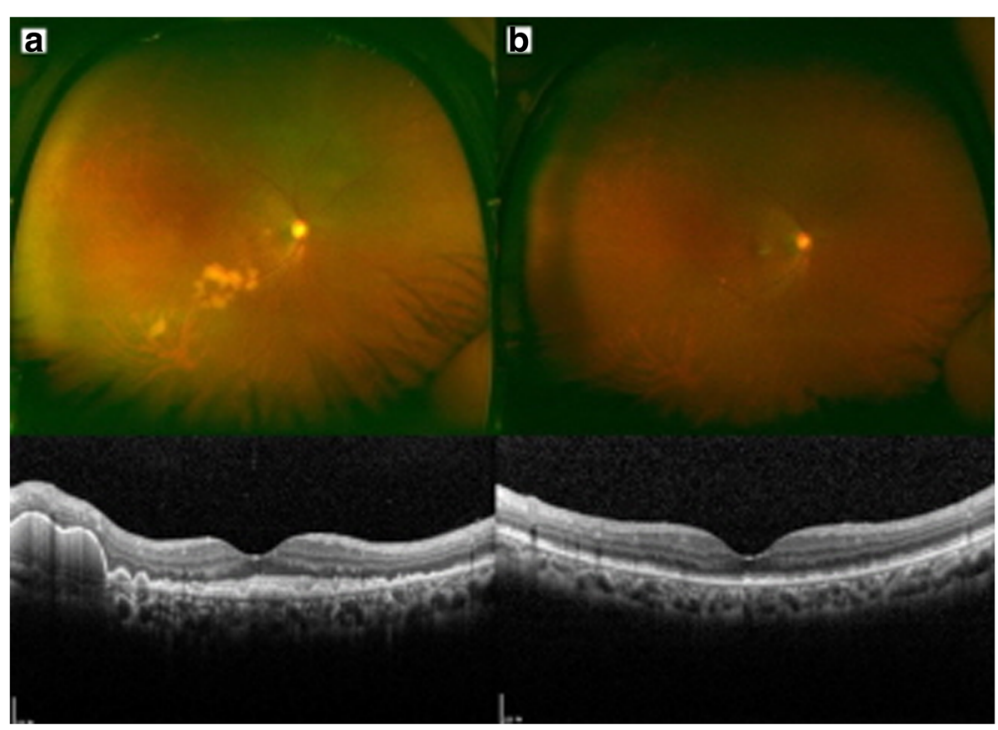

Fig. 2 Follow-up ophthalmologic findings in the right eye. a Two months after steroid treatment, yellow-white subretinal deposits had developed. The ellipsoid zone swelling area extended to the subfoveal area, and sub-retinal pigment epithelium (RPE) deposits were also increased. b Six months following intravitreal methotrexate treatment, subretinal and sub-RPE infiltration decreased, resulting in severe photoreceptor disruption 
eye (Fig. 1b). Fundus autofluorescence showed parafoveal granular hyperautofluorescence in both eyes (Fig. 1c).

Based on the negative results of various blood tests, she was diagnosed with noninfectious uveitis and started on oral prednisolone. She noticed mild improvement 1 month after treatment, but 2 months after treatment she showed a visual loss to 0.1 in her right eye. Compared with the initial visit, the swollen ellipsoid zone area extended under the fovea and subRPE yellow-white deposits were newly developed in the inferotemporal area (Fig. 2a). Also, mild vitreous opacity with haziness was noticed in the right eye. We suspected primary intraocular lymphoma (PIOL), and a 25-gauge microincision vitrectomy in right eye was performed. Vitreous cytology revealed atypical mononuclear cells with positive CD20 immunostaining. PCR of the vitreous fluid was negative for herpes and cytomegalovirus.

The patient was evaluated by an oncologist, and no lymphoma involvement in the brain was found. She was treated with high-dose systemic methotrexate as well as intravitreal methotrexate injections $(400 \mu \mathrm{g} / 0.1 \mathrm{~mL})$ twice weekly for 4 weeks for induction, once weekly for 8 weeks, and once monthly for 9 months in the right eye. Given the potential causal relationship between PIOL and anti-TNF $\alpha$ agents, etanercept was discontinued.

Three months after treatment, her right eye showed a decrease in subretinal infiltration and subRPE deposits, which further resolved at 6 months following treatment (Fig. 2b). Over the course, her left eye also showed gradual improvement in funduscopic examination as well as SD-OCT findings without intravitreal methotrexate injection, potentially due to systemic chemotherapy. Because of severe photoreceptor disruption, BCVA was 0.1 OD and 0.4 OS at the final visit.

\section{Discussion}

The risk of lymphoma is still a major concern in patients using anti-TNF $\alpha$ agents. The exact mechanisms underlying lymphomagenesis are unclear. However, considering several anticancer mechanisms of TNF, such as induction of tumor vessel obstruction and tumor immune surveillance, effects related to anti-TNF $\alpha$ agents may contribute to tumor development by suppressing the anticancer mechanisms of TNF [4].

Reported rates of cytological sensitivity and specificity for the diagnosis of primary vitreoretinal lymphoma (PVRL) vary widely, but cytology alone is able to confirm PVRL in $45-60 \%$ of cases, and false positive results are considered rare. Experienced technicians and ocular cytopathologists play a major role in this success rate $[5,6]$. In addition to the cytopathologic diagnosis, multicolor flow cytometry methods such as monoclonality can support $B$ or $T$ cell PVRL [7]. Either a B-cell population stains positively for B-cell markers (CD19, CD20, or CD22) with restricted expression of either $\mathrm{k}$ or $\lambda$, or a $\mathrm{T}$-cell population stains positively for T-cell markers (CD3, CD4). Furthermore, cytokine analysis of IL-10 and IL6 ratios greater than 1.0 are suggested diagnostic criteria for lymphoma [8-10]. Also, methods for detecting IgH gene rearrangement can serve as an additional diagnostic method $[11,12]$. In this case, because we lacked a laboratory setting, cytopathologic diagnosis was made microscopically. We noted atypical cytomorphology with CD20 positivity, suggestive of lymphoma. The gold standard is pathologic diagnosis for lymphoma, and pathologic confirmation was made through an experienced pathologist.

Because no standard therapy for PVRL has been established, the International Primary CNS Lymphoma Collaborative Group organized a symposium on PVRL in 2011. Their guidelines established local treatment [intra-vitreal methotrexate, intravitreal rituximab, or low-dose (30-35 Gy) stereotactic external beam radiotherapy to the eye] with close follow as the preferred treatment option for cases without CNS and systemic involvement. If both eyes are involved, systemic chemotherapy has been suggested in addition to intravitreal medications for bilateral PVRL [13, 14]. Clinically, pars plana vitrectomy is frequently performed for PVRL biopsy because its diagnostic yield is superior to that of vitreous aspirate. A 25-gauge transconjunctival sutureless vitrectomy is a safe and effective technique for diagnosing PVRL. Considering the recent trend of minimally invasive vitrectomy, 25-gauge vitrectomy is an appropriate diagnostic method for vitreous sampling and diagnosis $[15,16]$. In this case, suspicious bilateral involvement of PIOL, without CNS involvment with acutely developed in the unilateral eye, advocates the treatment of systemic chemotherapy treatment with intravitreal methotrexate injection and 25-gauge vitrectomy for diagnostic purposes.

Despite numerous systematic reviews and meta-analyses, there is insufficient evidence to establish a causal relationship between use of anti-TNF $\alpha$ agents and lymphomagenesis. The overall malignancy rate of anti-TNF $\alpha$ agents is reportedly 0.6 per 100 person-years, and the rate of etanercept is 10.47 per 1000 person-years [17]. The use of biologics among patients with RA, included in randomized controlled trials of at least 6 months' duration, was not significantly associated with an increased risk of malignancy compared with other disease-modifying antirheumatic drugs, or compared to placebo [18]. In another study, there was no difference in the risk of lymphoma for the TNF inhibitor versus the biological-naive group: hazard ratio (HR) 1.00 (95\% CI 
0.56 to 1.80). No differences in risk were observed for individual TNF inhibitors $[19,20]$. There is no evidence that tumor necrosis factor inhibition influences the risk of lymphoma, over background risk, in subjects with RA. However, there are case reports of primary intraocular B-cell lymphoma arising during methotrexate and tumor necrosis factor inhibitor treatment [21]. A direct relationship between primary intraocular lymphoma or PVRL and the use of biologic agents remains controversial; however, clinicians should be aware of the possibility of PIOL in patients with chronic refractory uveitis.

Our patient could represent a case of primary intraocular lymphoma, possibly related to the use of the anti-TNF $\alpha$ agent etanercept. A diagnosis of intraocular lymphoma should be suspected when patients with underlying rheumatologic disease develop chronic refractory uveitis during anti-TNF $\alpha$ treatment.

\section{Conclusions}

Ophthalmologists should be aware of the relationship between immunosuppression via anti-TNF $\alpha$ medication, and an increased risk for lymphoma, especially in patients with underlying rheumatologic disorders, and especially in cases with suspected chronic refractory uveitis. Further evidence is needed pertaining to cancer risk in patients who are using, or have used, anti-TNF $\alpha$ agents.

\section{Abbreviations}

BCVA: Best corrected visual acuity; EZ: Ellipsoid zone; PCR: Polymerase chain reaction; PIOL: Primary intraocular lymphoma; PVRL: Primary vitreoretinal lymphoma; RA: Rheumatoid arthritis; RPE: Retinal pigment epithelium; SDOCT: Spectralis-domain optical coherence tomography; TNF: Tumor necrosis factor

\section{Availability of data and materials}

All data that supports these findings are contained within this manuscript.

\section{Authors' contributions}

Collection of data and preparation of the manuscript (SWK, ARC, YHY), Study design and concept, supervision and critical revision of the manuscript (YHY). All authors had full access to all the data in the study and take responsibility for the integrity of the data and the accuracy of the data analysis. All authors read and approved the final manuscript.

\section{Ethics approval and consent to participate}

This study complied with the tenets of the Declaration of Helsinki. This report was determined to be exempt from IRB review by the Institutional Review Board of Asan Medical Center.

\section{Consent for publication}

Informed consent was obtained from the patient for publication.

\section{Competing interests}

The Authors declare that they have no competing interests.
Received: 4 April 2018 Accepted: 20 June 2018

Published online: 28 June 2018

\section{References}

1. Baecklund E, lliadou A, Askling J, Ekbom A, Backlin C, Granath F, Catrina Al, et al. Association of chronic inflammation, not its treatment, with increased lymphoma risk in rheumatoid arthritis. Arthritis Rheum. 2006;54:692-701.

2. Bongartz T, Sutton AJ, Sweeting MJ, Buchan I, Matteson EL, Montori V. AntiTNF antibody therapy in rheumatoid arthritis and the risk of serious infections and malignancies: systematic review and meta-analysis of rare harmful effects in randomized controlled trials. JAMA. 2006:295:2275-85.

3. Georgakopoulou EA, Andreadis D, Arvanitidis E, Loumou P. Biologic agents and oral diseases-an update on clinical applications. Acta Dermatovenerol Croat. 2013;21:24-34.

4. Mocellin S, Pilati P, Nitti D. Towards the development of tumor necrosis factor (TNF) sensitizers: making TNF work against cancer. Curr Pharm Des. 2007;1:537-51

5. Davis JL, Viciana AL, Ruiz P. Diagnosis of intraocular lymphoma by flow cytometry. Am J Ophthalmol. 1997;124:362-72.

6. Kimura K, Usui Y, Goto H. Japanese intraocular lymphoma study group. Clinical features and diagnostic significance of the intraocular fluid of 217 patients with intraocular lymphoma. Jpn J Ophthalmol. 2012;56:383-9.

7. Missotten T, Tielemans D, Bromberg JE, et al. Multicolor flowcytometric immunophenotyping is a valuable tool for detection of intraocular lymphoma. Ophthalmology. 2013;120:991-6.

8. Cassoux N, Giron A, Bodaghi B, et al. IL-10 measurement in aqueous humor for screening patients with suspicion of primary intraocular lymphoma. Invest Ophthalmol Vis Sci. 2007;48:3252-9.

9. Costopoulos M, Touitou V, Golmard JL, et al. ISOLD: a new highly sensitive interleukin score for intraocular lymphoma diagnosis. Ophthalmology. 2016; 123:1626-8.

10. Mehta M, Rasheed RA, Duker J, et al. Vitreous evaluation: a diagnostic challenge. Ophthalmology. 2015;122:531-7.

11. Langerak AW, Groenen PJ, Bruggemann M, et al. EuroClonality/BIOMED-2 guidelines for interpretation and reporting of lg/TCR clonality testing in suspected lymphoproliferations. Leukemia. 2012;26:2159-71.

12. Coupland SE, Hummel M, Muller HH, et al. Molecular analysis of immunoglobulin genes in primary intraocular lymphoma. Invest Ophthalmol Vis Sci. 2005;46:3507-14.

13. Chan CC, Rubenstein JL, Coupland SE, et al. Primary vitreoretinal lymphoma: a report from an international primary central nervous system lymphoma collaborative group symposium. Oncologist. 2011;16:1589-99.

14. Peer J, Hochberg FH, Foster CS. Clinical review: treatment of vitreoretinal Iymphoma. Ocul Immunol Inflamm. 2009;17:299-306.

15. Gonzales JA, Chan CC. Biopsy techniques and yields in diagnosing primary intraocular lymphoma. Int Ophthalmol. 2007:27:241-50.

16. Yeh $S$, Weichel ED, Faia $L$, et al. 25-gauge transconjunctival sutureless vitrectomy for the diagnosis of intraocular lymphoma. Br J Ophthalmol. 2010; 94:633-8.

17. Kievit W, Fransen J, Adang EM, den Broeder AA, Bernelot Moens HJ, Visser $\mathrm{H}$, et al. Long-term effectiveness and safety of TNF-blocking agents in daily clinical practice: results from the Dutch rheumatoid arthritis monitoring register. Rheumatology (Oxford). 2011;50:196-203.

18. Mercer LK, et al. Risk of lymphoma in patients exposed to antitumour necrosis factor therapy: results from the British Society for Rheumatology biologics register for rheumatoid arthritis. Ann Rheum Dis. 2017 Mar;76(3): 497-503.

19. Bongartz T, Sutton AJ, Sweeting MJ, et al. Anti-TNF antibody therapy in rheumatoid arthritis and the risk of serious infections and malignancies: systematic review and meta-analysis of rare harmful effects in randomized controlled trials. JAMA. 2006;295(19):2275-85.

20. Lopez-Olivo MA, Tayar JH, Martinez-Lopez JA, et al. Risk of malignancies in patients with rheumatoid arthritis treated with biologic therapy: a metaanalysis. JAMA. 2012;308(9):898-908.

21. Nagata K, Inaba T, Kinoshita S. CD5-positive primary intraocular B-cell lymphoma arising during methotrexate and tumor necrosis factor inhibitor treatment. Case Rep Ophthalmol. 2015;6(3):301-6.

\section{Publisher's Note}

Springer Nature remains neutral with regard to jurisdictional claims in published maps and institutional affiliations. 